\title{
Influence of the Rotational Alignment of the Femoral and Patellar Components on Patellar Tilt in Total Knee Arthroplasty
}

\author{
Jong Heon Kim, MD, Byeong-Wook Yoo, MD, and Chul-Woong Kim, MD \\ Department of Orthopedic Surgery, Guri Hospital, Hanyang University College of Medicine, Guri, Korea
}

\begin{abstract}
Purpose: The purpose of this study was to analyze the influence of rotational alignment of the femoral and patellar components on patellar tilt after total knee arthroplasty (TKA).

Materials and Methods: A total of 56 patients (76 knees) who underwent TKA using Advance Medial Pivot Knee system between May 2009 and April 2011 and were available for minimum 1-year follow-up were enrolled in this study. Whiteside's line and the transepicondylar line were used to determine the rotational alignment of the femoral component. Patella cut was aimed to be parallel to the anterior patellar cortex during surgery. Radiographic evaluation was performed using plain axial radiographs. The rotational alignment of the femoral component was measured as the angle between the anterior condylar axis and the surgical transepicondylar axis. The patellar resection angle was measured between the patellar resection axis and the anterior cortical line of the patella. Patellar tilt was evaluated to investigate the correlation with the rotation of the femoral component and patellar resection angle.

Results: The mean rotation of the femoral component was $0.42^{\circ} \pm 3.18^{\circ}$ of internal rotation. The mean patellar resection angle was $1.82^{\circ} \pm 3.44^{\circ}$, indicating medial overresection. The mean patellar tilt was $6.12^{\circ} \pm 4.31^{\circ}$ of lateral tilt. The rotational angle of the femoral component showed a negative correlation with patellar tilt in the linear regression analysis $(\mathrm{p}=0.749)$, but the patellar resection angle showed a positive correlation with patellar tilt $(\mathrm{p}<0.001)$.

Conclusions: Accurate patellar resection is recommended for proper patellar tracking in TKA.
\end{abstract}

Keywords: Knee, Arthroplasty, Component roation, Patellar tracking

\section{Introduction}

Patellar maltracking after total knee arthroplasty (TKA) has been associated with anterior knee pain and limited range of motion, which would eventually result in impaired functional recovery and development of severe complications such as implant wear/loosening and patellar fracture ${ }^{1-3)}$. A variety of studies

Received September 21, 2014; Revised (1st) December 27, 2014;

(2nd) May 25, 2015; Accepted June 16, 2015

Correspondence to: Jong Heon Kim, MD

Department of Orthopedic Surgery, Guri Hospital, Hanyang University

College of Medicine, 153 Gyeongchun-ro, Guri 11923, Korea

Tel: +82-31-560-2313, Fax: +82-31-557-8781

E-mail: jhkim111@hanyang.ac.kr

This is an Open Access article distributed under the terms of the Creative Commons Attribution Non-Commercial License (http://creativecommons.org/licenses/by-nc/4.0/) which permits unrestricted non-commercial use, distribution, and reproduction in any medium, provided the original work is properly cited. have suggested factors that could be linked with patellar tracking. Some cadaveric studies analyzing the relative impact of knee components demonstrated that the patellar resection angle, location of the patellar component, and rotational alignment of the femoral component could affect postoperative patellar tracking ${ }^{4,5}$. In an in vivo study, Kawano et al. ${ }^{6}$ reported the location of the patellar component, patellar resection angle, and lateral retinacular release as related factors. Some authors associated patellar tracking with preoperative patellar tilt ${ }^{7)}$, surgical approach ${ }^{8)}$, location and rotational alignment of the implant ${ }^{9-12)}$, and implant design and soft tissue balance ${ }^{13,14)}$. However, there is still controversy on which of these factors should be taken into consideration during surgery; the abovementioned factors may interact during surgery, and studies on relative impact of each factor using standardized methods are lacking.

Accordingly, we designed this study to elucidate the influence of the two possible determinants of patellar tracking, the rotational 
alignment of the femoral component ${ }^{10-12)}$ and patellar resection angle ${ }^{4,6)}$, on patellar tilt among patients who underwent TKA in the same surgical setting.

\section{Materials and Methods}

Of the patients who underwent TKA using Advance Medial Pivot Knee system (Wright Medical Technology Inc., Arlington, TN, USA) between May 2009 and April 2011 and who were available for more than 1-year follow-up, a total of 56 patients (76 knees) were included in this retrospective study. The exclusion criteria were revision TKA, severe patellar deformity, preoperative subluxation or dislocation of the patella, severe valgus deformity of the lower limb, TKA without patellar resurfacing, and lateral retinacular release in TKA. There were 11 males and 45 females, and 20 of them underwent bilateral TKA. Their mean age was $68 \pm 7.4$ years. The indication for surgery was osteoarthritis in 71 knees, rheumatoid arthritis in 3 knees, and osteonecrosis in 2 knees. The mean preoperative lower limb alignment was $3.16^{\circ}$ varus (range, $7.6^{\circ}$ valgus to $14.1^{\circ}$ varus). The mean preoperative patellar tilt was $1.83^{\circ}$ (range, $2.5^{\circ}$ of medial tilt to $10.4^{\circ}$ of lateral tilt). In all knees, the posterior cruciate ligament-sacrificing Advance Medial Pivot Knee system was inserted in TKA performed by the same surgeon using the following surgical procedure.

The surgery was carried out through a midline skin incision using the medial parapatellar approach in all patients under tourniquet control. Medial soft tissue release was additionally performed if considered necessary based on the medial/lateral flexion-extension gap assessment during surgery. During implant insertion, care was taken to maintain approximately $3^{\circ}$ external rotation of the femoral component with respect to the posterior condylar axis, and further adjustments were made to align the femoral component parallel to the surgical transepicondylar axis as much as possible and perpendicular to the anteroposterior axis (Whiteside's line). The center of the femoral component was aligned with the trochlear groove. The rotational alignment of the tibial component was determined by a line connecting the medial $1 / 3$ of the tibial tubercle and the center of the posterior cruciate ligament. Upon confirming with a rod the tibial component was aligned with a line passing though the tibial crest and the center of the ankle joint, component insertion was done. For patellar resurfacing, the lateral margin of the patella was exposed and the overall patellar thickness was measured using a caliper. Using the free-handle technique, patellar resection was performed parallel to the anterior cortex, taking care to produce symmetric thickness at the medial and lateral facets of the patella. The longi- tudinal axis of the dome-shaped patellar component was aligned with the longitudinal axis at the central ridge of the patella, and the bone-prosthesis composite thickness was intended to be equal to the original thickness of the patella.

Radiographic assessment was performed using the 1-year followup axial plain radiographs (merchant view). The rotational alignment of the femoral component was measured according to the method of Kanekasu et al. ${ }^{15}$ : the angle subtended by the transepicondylar axis and the anterior condylar axis (femoral component rotation angle) was measured, and external rotation was expressed as a positive value (Fig. 1). The rotational alignment of the patellar component was assessed according to the method of Chan and Gill ${ }^{7}$. Two perpendicular lines were drawn at the medial and lateral facets of the patella. A line connecting the ends of the two perpendicular lines was drawn at the anterior patellar cortex. The angle subtended by the line at the anterior patellar cortex and a line tangent to the resected surface of the patellar component (patellar resection angle) was measured, and medial over-resection was assigned a positive value (Fig. 1). Patellar tilt was measured according to the method of Gomes et al. ${ }^{16}$ : the angle between the anterior condylar axis and the resected surface of the patellar component was measured (patellar tilt angle), and lateral tilt was given a positive value (Fig. 1).

Statistical analysis was done using SAS ver. 9.2 (SAS Institute Inc., Cary, NC, USA) to compare the influence of the femoral

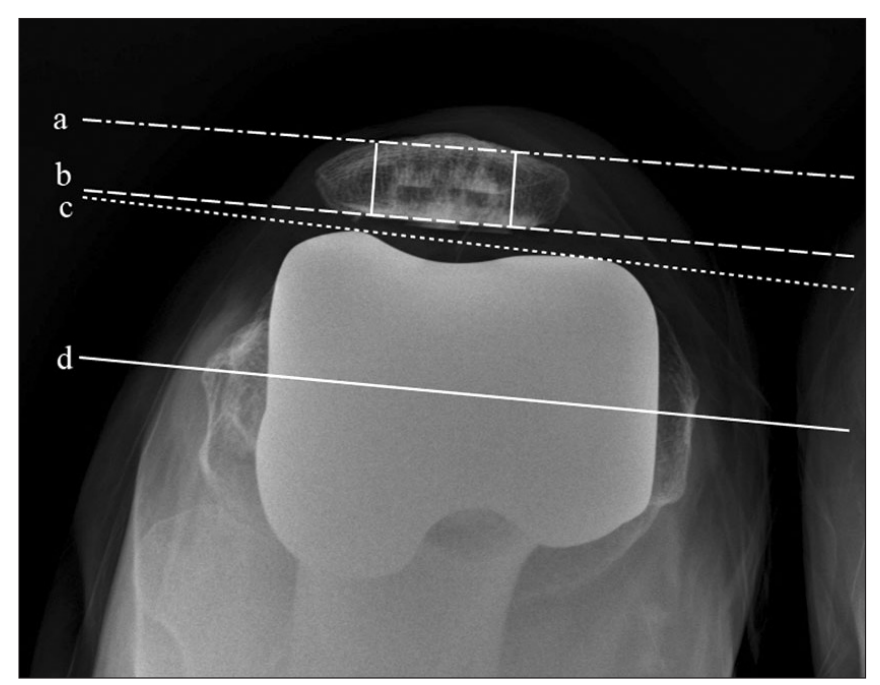

Fig. 1. The patellar resection angle (a-b), patellar tilt $(\mathrm{b}-\mathrm{c})$, and femoral component rotation angle $(\mathrm{c}-\mathrm{d})$ were measured on plain axial radiographs. a: a line at the anterior patellar cortex connecting the ends of perpendicular lines drawn at the medial and lateral aspects of the patella, b: patellar resection axis, c: anterior condylar axis, d: surgical transepicondylar axis. 


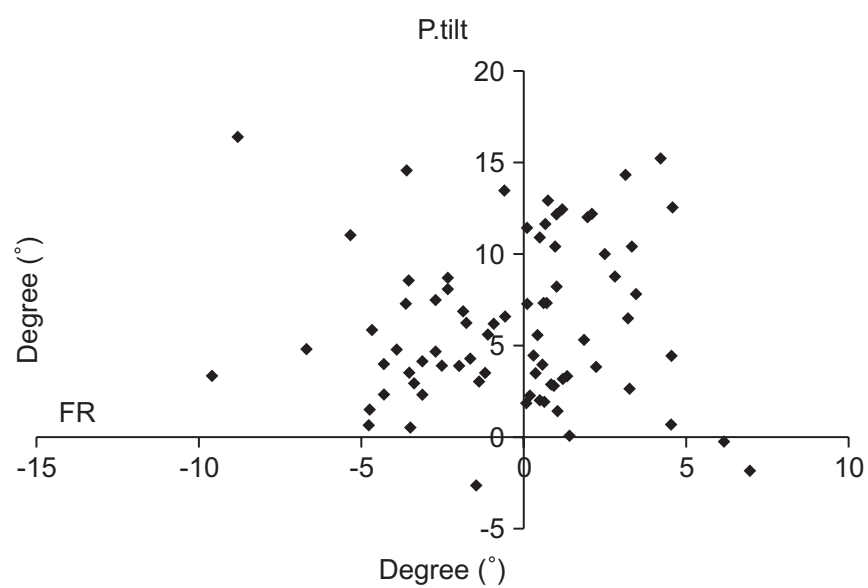

Fig. 2. The graph shows no positive correlation between patella tilt (P.tilt) and femoral component rotation angle (FR) $(\mathrm{p}=0.749)$.

component rotation angle and patellar resection angle on patellar tilt. Cronbach' alpha test was used to evaluate the reliability of the measurements performed twice by a single observer. The influence of the two parameters on patellar tilt was evaluated using multivariate regression analysis.

\section{Results}

The mean femoral component rotation angle was $0.42^{\circ} \pm 3.18^{\circ}$ of internal rotation. The mean patellar resection angle was $1.82^{\circ} \pm 3.4^{\circ}$, indicating medial over-resection. The mean patellar tilt was $6.12^{\circ} \pm 4.31^{\circ}$, indicating lateral tilting. There was no statistically significant relationship between the femoral component rotation angle and patellar tilt ( $\mathrm{p}=0.749$ ) (Fig. 2); however, the patellar resection angle showed a significant correlation with patellar tilt ( $\mathrm{p}<0.001, \mathrm{r}=0.289$ ) (Fig. 3). The Cronbach' alpha value was $\geq 0.7$, indicating good intraobserver reliability.

\section{Discussion}

In this study, we analyzed the influence of the rotational alignment of the femoral component and patellar resection angle on patellar tilt using plain radiographs (Merchant view) in patients who received the same knee implant in TKA performed by the same surgeon to ensure consistent surgical setting. Based on the radiographic assessment, we believe that patellar resection angle has more significant influence than the rotational alignment of the femoral component on patellar tilt.

Independent influence of factors associated with patellar tracking can be assessed through simulation in cadaveric studies, and there are in vivo studies reporting on the possible contribut-

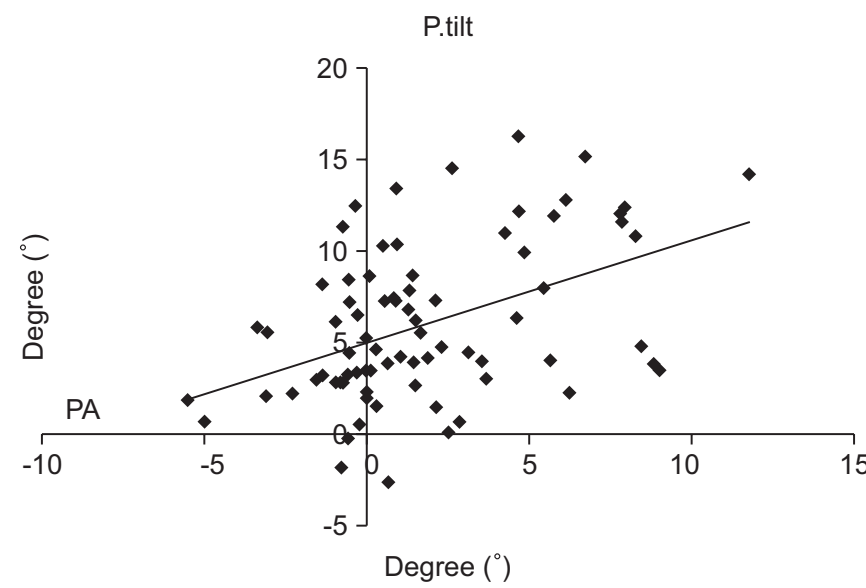

Fig. 3. The graph shows a positive correlation between patella tilt (P.tilt) and patellar resection angle $(\mathrm{PA})(\mathrm{R}=0.289, \mathrm{p}=0.019)$.

ing factors; however, their results have failed to generate broad consensus due to the lack of standardization. In this study, we attempted to investigate the influence of factors that have been related to patellar tilt, such as the rotational alignment of the femoral component ${ }^{10-12)}$ and patellar resection angle $e^{4,6)}$, in patients who underwent TKA according to a standardized surgical procedure at our institution, and compare our results with those in the previous literature.

Kanekasu et al. ${ }^{15)}$ compared the preoperative axial radiographs and computed tomography (CT) scans in the assessment of the rotational alignment of the femoral component: there was no remarkable discrepancy with regard to the posterior condylar axis of the femur and clinical transepicondylar axis (a line connecting the prominences of the medial and lateral epicondyles) between the two imaging modalities. Thus, they concluded that axial radiography could be useful for the assessment of the rotational alignment of the femoral component in TKA patients. In the current study, under the hypothesis that the anterior condylar axis and the posterior condylar axis form a constant angle, we used the surgical transepicondylar axis (a line connecting the sulcus of the medial epicondyle and the lateral epicondylar prominence) and the anterior condylar axis of the femoral component to assess the rotational alignment of the femoral component (Fig. 1).

The mean femoral component rotation angle was $0.42^{\circ} \pm 3.18^{\circ}$ of internal rotation, which may be attributable to the design characteristics of the Advance Medial Pivot Knee system where the lateral margin of the patellar groove is elevated.

Chan and Gill ${ }^{7)}$ reported that over-resection of the patella is a common occurrence because the flat or slightly convex medial patellar articular surface is thicker than the concave lateral surface. On the other hand, Baldini et al. ${ }^{17)}$ suggested that misuse of 
instruments and errors of free-handle technique often result in medial over-resection. In the current study, we aimed to perform symmetrical patellar resection with reference to the anterior patellar cortex; however, the mean patellar resection angle was $1.82^{\circ} \pm 3.44^{\circ}$, indicating medial over-resection, which could be explained by the observations described in the abovementioned studies.

The relationship between the rotational alignment of the femoral component and patellar lateral tilt has been addressed in various studies. Matsuda et al.9 reported that the mean femoral component rotation angle was $6.1^{\circ}$ of internal rotation (range, $2.7^{\circ}$ to $11.2^{\circ}$ ) and the mean tibial component rotation was $16.7^{\circ}$ of internal rotation (range, $2.4^{\circ}$ to $27.7^{\circ}$ ) on CT scans taken with the knee in $30^{\circ}$ flexion. The study also showed a significant positive correlation between patellar tilt and the sum of the internal rotation angles. Berger et al. ${ }^{2)}$ observed significantly greater incidence of patellofemoral complications, such as excessive patellar lateral tilt, in the group with excessive combined internal rotation of the femoral and tibial components after TKA $\left(n=30\right.$; range, $1^{\circ}$ of external rotation to $15^{\circ}$ of internal rotation) than the control group (range, $0^{\circ}$ to $10^{\circ}$ of external rotation). However, in the current study, no statistically significant correlation was found between the femoral component rotation angle and patellar tilt ( $\mathrm{p}=0.749$, Fig. 2). This may be attributable to the fact that the degree of femoral component internal rotation was relatively small in the patients compared to that in previous studies where greater internal rotations of the femoral and tibial components were present, and we attempted to have the tibial component placed in a neutral position.

On the relationship between the patellar resection angle and patellar tilt, Chan and Gill ${ }^{7)}$ reported that an extensive medial patellar resection would result in a great lateral patellar tilt. Their result was in agreement with the study of Kawano et al. ${ }^{6}$. Approximately $5^{\circ}$ of lateral patellar tilt is normal in a healthy knee in $60^{\circ}$ flexion $^{18)}$; therefore, $5^{\circ}$ of patellar tilt would improve the congruence between the femoral and patellar components. On the other hand, Baldini et al. ${ }^{17)}$ reported that anterior pain was more prominent in the group with asymmetric patellar resection than in the group with symmetric resection. Anglin et al. ${ }^{4)}$ advised against excessively asymmetric patellar resection because improper patellar resection results in patellar shift. In the current study, we observed a positive correlation between the patellar resection angle and patellar tilt $(\mathrm{p}<0.001, \mathrm{r}=0.289$; Fig. 3 ), which also implies a positive correlation between medial over-resection and lateral tilt as shown in previous studies.

The limitations of our study include 1) the small study popula- tion, 2) lack of clinical assessment on the postoperative outcome, 3) exclusion of $\mathrm{CT}$ in the assessment of femoral component rotation and of some factors that could influence patellar tilt in spite of our efforts to ensure standardization utilizing the same femoral component and to place the femoral component at a proper external rotation in the original trochlear groove and the patellar component in neutral rotation in the original position. Therefore, various factors that were not addressed in this study should be included in further research.

Patella maltracking after TKA can lead to the development of various complications including anterior knee pain, limited range of motion, asymmetrical wear and loosening, and patellar fracture. Therefore, proper patellar tracking is essential for successful $\mathrm{TKA}^{1,4,19,20)}$. In the current study, we investigated the influence of the rotational alignment of the femoral component and patellar resection angle on patellar tilt that could affect patellar tracking. The results showed that patellar resection angle has greater influence on patellar tilt. For consistent patellar resection, we recommend the free-handle technique in TKA should be used 1) by referring to the preoperative radiographic findings on the shape and location of the patella, 2) taking care to avoid excessive medial resection, and 3) aiming to perform multiple piecemeal resections based on intraoperative assessment. It is also advised to assess patellar tracking during surgery with a trial patellar component placed in the knee. Ledger et al. ${ }^{20)}$ suggested the medial and lateral edges of the quadriceps tendon and lateral edge of the patellar tendon as anatomical landmarks for consistent patellar resection. Belvedere et al. ${ }^{21)}$ advocated the efficacy of surgical navigation system for patellar resection. It is our understanding that further research and efforts are needed to improve consistency in patellar resection.

\section{Conclusions}

The patellar resection angle had greater influence on patellar tilt than the rotational alignment of the femoral component in patients who underwent TKA performed in a consistent surgical setting. Therefore, accurate patellar resection is recommended for achieving proper patellar tracking after TKA.

\section{Conflict of Interest}

No potential conflict of interest relevant to this article was reported. 


\section{References}

1. Bindelglass DF, Cohen JL, Dorr LD. Patellar tilt and subluxation in total knee arthroplasty. Relationship to pain, fixation, and design. Clin Orthop Relat Res. 1993;(286):103-9.

2. Berger RA, Crossett LS, Jacobs JJ, Rubash HE. Malrotation causing patellofemoral complications after total knee arthroplasty. Clin Orthop Relat Res. 1998;(356):144-53.

3. Lombardi AV Jr, Engh GA, Volz RG, Albrigo JL, Brainard BJ. Fracture/dissociation of the polyethylene in metal-backed patellar components in total knee arthroplasty. J Bone Joint Surg Am. 1988;70:675-9.

4. Anglin C, Brimacombe JM, Hodgson AJ, Masri BA, Greidanus NV, Tonetti J, Wilson DR. Determinants of patellar tracking in total knee arthroplasty. Clin Biomech (Bristol, Avon). 2008;23:900-10.

5. Miller MC, Berger RA, Petrella AJ, Karmas A, Rubash HE. Optimizing femoral component rotation in total knee arthroplasty. Clin Orthop Relat Res. 2001;(392):38-45.

6. Kawano T, Miura H, Nagamine R, Urabe K, Matsuda S, Mawatari T, Moro-Oka T, Iwamoto Y. Factors affecting patellar tracking after total knee arthroplasty. J Arthroplasty. 2002;17:942-7.

7. Chan KC, Gill GS. Postoperative patellar tilt in total knee arthroplasty. J Arthroplasty. 1999;14:300-4.

8. Komatsu T, Ishibashi Y, Otsuka H, Nagao A, Toh S. The effect of surgical approaches and tourniquet application on patellofemoral tracking in total knee arthroplasty. J Arthroplasty. 2003;18:308-12.

9. Matsuda S, Miura H, Nagamine R, Urabe K, Hirata G, Iwamoto Y. Effect of femoral and tibial component position on patellar tracking following total knee arthroplasty: 10-year follow-up of Miller-Galante I knees. Am J Knee Surg. 2001; 14:152-6.

10. Akagi M, Matsusue Y, Mata T, Asada Y, Horiguchi M, Iida H, Nakamura T. Effect of rotational alignment on patellar tracking in total knee arthroplasty. Clin Orthop Relat Res. 1999;(366):155-63.

11. Jung JW, Cheon SH, Kyung HS. The correlation of postoper- ative femoral component rotation angle and patella tilt angle with clinical results in total knee arthroplasty. J Korean Knee Soc. 2011;23:96-103.

12. Verlinden C, Uvin P, Labey L, Luyckx JP, Bellemans J, Vandenneucker $\mathrm{H}$. The influence of malrotation of the femoral component in total knee replacement on the mechanics of patellofemoral contact during gait: an in vitro biomechanical study. J Bone Joint Surg Br. 2010;92:737-42.

13. Varadarajan KM, Rubash HE, Li G. Are current total knee arthroplasty implants designed to restore normal trochlear groove anatomy? J Arthroplasty. 2011;26:274-81.

14. Indelli PF, Marcucci M, Cariello D, Poli P, Innocenti M. Contemporary femoral designs in total knee arthroplasty: effects on the patello-femoral congruence. Int Orthop. 2012; 36:1167-73.

15. Kanekasu K, Kondo M, Kadoya Y. Axial radiography of the distal femur to assess rotational alignment in total knee arthroplasty. Clin Orthop Relat Res. 2005;(434):193-7.

16. Gomes LS, Bechtold JE, Gustilo RB. Patellar prosthesis positioning in total knee arthroplasty. A roentgenographic study. Clin Orthop Relat Res. 1988;(236):72-81.

17. Baldini A, Anderson JA, Cerulli-Mariani P, Kalyvas J, Pavlov H, Sculco TP. Patellofemoral evaluation after total knee arthroplasty: validation of a new weight-bearing axial radiographic view. J Bone Joint Surg Am. 2007;89:1810-7.

18. Nagamine R, Otani T, White SE, McCarthy DS, Whiteside LA. Patellar tracking measurement in the normal knee. J Orthop Res. 1995;13:115-22.

19. Barrack RL, Burak C. Patella in total knee arthroplasty. Clin Orthop Relat Res. 2001;(389):62-73.

20. Ledger M, Shakespeare D, Scaddan M. Accuracy of patellar resection in total knee replacement. A study using the medial pivot knee. Knee. 2005;12:13-9.

21. Belvedere C, Ensini A, Leardini A, Dedda V, Feliciangeli A, Cenni F, Timoncini A, Barbadoro P, Giannini S. Tibio-femoral and patello-femoral joint kinematics during navigated total knee arthroplasty with patellar resurfacing. Knee Surg Sports Traumatol Arthrosc. 2014;22:1719-27. 